\title{
Desempenho de Bezerros de Corte Filhos de Vacas Submetidas a Diferentes Manejos Alimentares, Desmamados aos 42 ou 63 Dias de Idade
}

\author{
Liliane Cerdótes $^{1}$, João Restle ${ }^{2}$, Dari Celestino Alves Filho ${ }^{3}$, Paulo Santana Pacheco ${ }^{4}$, \\ Régis Luis Missio ${ }^{5}$, Fábio Cervo Garagorry ${ }^{6}$
}

\begin{abstract}
RESUMO - O objetivo deste trabalho foi avaliar o desempenho de bezerros de corte, do nascimento aos 12 meses de idade, filhos de vacas Charolês (C), Nelore (N), mestiças CN e NC, mantidas em pastagem nativa, suplementadas com farelo de arroz integral e que desmamaram aos 42 ou 63 dias pós-parto, ou não suplementadas e que desmamaram aos 63 dias. A idade das vacas variou de 3 a 12 anos, sendo agrupadas em quatro classes, primíparas, jovens, adultas e velhas. Bezerros desmamados aos 63 dias de idade, cujas vacas foram suplementadas, apresentaram maior ganho de peso médio diário (GMD) durante o aleitamento (561 contra 457 g), foram mais pesados ao desmame (67 contra 59,9 kg) e aos 84 dias (76 contra 69,2 kg) em relação aqueles cujas vacas não foram suplementadas, não diferindo destes nos pesos aos 5, 7 e 12 meses. Bezerros desmamados aos 42 dias apresentaram menor GMD nos primeiros 21 dias após o desmame (360 contra 482 g) e foram menos pesados aos 12 meses (174,4 contra 189,4 kg) em relação aos desmamados aos 63 dias. Bezerros mestiços filhos de vacas $\mathrm{CN}$ e NC foram mais pesados ao desmame que os puros, não diferindo dos C aos 12 meses. Bezerros $\mathrm{N}$ apresentaram peso similar aos $\mathrm{C}$ no desmame, mas foram mais leves e apresentaram condição corporal inferior do que os últimos aos 5, 7 e 12 meses. O peso e a condição corporal dos bezerros filhos de vacas primíparas foi inferior aos demais, no entanto, não diferiram destes aos 9 e 12 meses. Não se justifica a suplementação das vacas quando se considera o peso dos bezerros após os 5 meses. O desmame aos 42 dias de idade não é recomendado, pois resulta em menor peso aos 12 meses.
\end{abstract}

Palavras-chave: Bos indicus, Bos taurus, cruzamento, estado corporal, desmame precoce, ganho de peso

\section{Performance of Beef Calves Produced by Cows Submitted to Different Feeding Managements, Weaned at 42 or 63 Days of Age}

\begin{abstract}
The objective of this work was to evaluate the performance of beef calves, from birth to weaning, produced by Charolais (C), Nellore (N), CN and NC crossbred cows, kept on native pasture, supplemented with rice bran and weaned at 42 or 63 days postpartum, or non supplemented and weaned at 63 days. Cow age ranged from 3 to 12 years, being classified as: first calf, young, adult and old cows. Calves weaned at 63 days of age, whose cows were supplemented showed higher average daily weight gain (ADG) during lactation ( 561 vs. $457 \mathrm{~g}$ ), were heavier at weaning ( $67 \mathrm{vs.} 59.9 \mathrm{~kg}$ ) and at 84 days (76 vs. 69,2 kg) in relation to those whose cows were not supplemented, and did not differ in weight from the last ones at 5, 7 and 12 months. Calves weaned at 42 days showed lower ADG during the first 21 days after weaning ( $360 \mathrm{vs.} 482 \mathrm{~g}$ ) and were lighter at 12 months ( $174.4 \mathrm{vs} .189 .4 \mathrm{~kg}$ ) in relation to those weaned at 63 days. Crossbred calves produced by CN and NC crossbred cows were heavier at weaning then the straightbreds, not differing from the $\mathrm{C}$ calves at 12 months. Nellore calves were similar in weight to the $\mathrm{C}$ calves at weaning, but were lighter and displayed lower body condition score than the $\mathrm{C}$ at 5, 7 and 12 months. Calves produced by first calving cows were lighter and displayed lower body condition score than the other calves, however, not differing from the last ones at 9 and 12 months. Supplementation of cows is not justified when calf weight after 5 months is considered. Weaning calves at 42 days is not recommended because it results in lower weight at 12 months.
\end{abstract}

Key Words: Bos indicus, Bos taurus, crossbreeding, body condition, early weaning, weight gain

\section{Introdução}

O desmame definitivo realizado aos 90 dias surgiu na década de 80 como alternativa para melhorar a condição corporal (CC) das vacas e, conseqüentemente, o desempenho reprodutivo do rebanho de cria, quando mantido em condições de pastagem nativa (PN), no Rio Grande do Sul (Cachapuz, 1991; Moojen et al., 1994; Restle et al., 1999). No entanto, esta técnica beneficia principalmente as vacas que parem na primeira metade do período de parição (Restle \& Vaz, 1998). Mais recentemente, a idade ao desmame

\footnotetext{
${ }^{1}$ Zootecnista, doutoranda do curso de Pós-Graduação em Zootecnia da FCAV/UNESP. E.mail: cerdotes@fcav.unesp.br

2 Engenheiro-Agrônomo, PhD, Pesquisador Visitante/CNPq - Departamento de Produção Animal - UFG. E.mail: jorestle@terra.com.br

${ }^{3}$ Engenheiro-Agronômo, MS, Prof. Assistente do Depto. de Zootecnia da UFSM. E.mail: dcafilho@terra.com.br

${ }^{4}$ Zootecnista, aluno do curso de Pós-Graduação em Zootecnia da UFSM. E.mail: pspacheco@zipmail.com.br

${ }^{5}$ Aluno do curso de graduação em Zootecnia da UFSM. E.mail: regisluismissio@bol.com.br

${ }^{6}$ Aluno do curso de Agronomia da UFSM. E.mail: fgaragorry@pop.com.br
} 
foi reduzida para menos de 90 dias, visando aumentar o período entre o desmame e o final da época de reprodução, dando as vacas maior tempo para ciclarem e conceberem (Pascoal \& Vaz, 1997).

Se por um lado o desmame antecipado favorece o desempenho da vaca, o problema passa a ser o bezerro que deverá receber atenção especial para não comprometer seu desempenho futuro. Entre os fatores que afetam o peso do bezerro ao desmame, pode-se citar principalmente o peso com que esse é realizado e o nível nutricional pré-desmame, o qual é altamente dependente da produção de leite da mãe, nos primeiros meses de vida, e ainda o potencial genético dos bezerros e as interações entre estes fatores (Jenkins et al., 1991).

A produção de leite da vaca é diretamente afetada pelo nível nutricional a que está submetida no período pré e pós-parto e também pelo grupo genético e idade da vaca (Ribeiro \& Restle, 1991; Senna, 1996; Cerdótes et al., 2004).

Restle et al. (1999) verificaram que o ganho de peso de bezerros desmamados aos três meses de idade foi mais baixo até os sete meses do que os mantidos ao pé da vaca até essa idade. Entretanto, devido ao maior ganho de peso após os sete meses, os bezerros desmamados antecipadamente não diferiram dos desmamados aos sete meses no peso aos 12, aos 18 e aos 24 meses de idade.

Tendo em vista a possibilidade de grandes benefícios com relação ao desempenho reprodutivo da vaca, este trabalho foi conduzido com o objetivo de avaliar o efeito do desmame aos 42 ou 63 dias de idade, associado ou não à suplementação das vacas mantidas em PN, no desempenho futuro dos bezerros.

\section{Material e Métodos}

O experimento foi conduzido no Setor de Bovinocultura de Corte do Departamento de Zootecnia da Universidade Federal de Santa Maria, Rio Grande do Sul. Foram utilizadas 159 vacas e bezerros, sendo 29 vacas da raça Charolês (C), 22 da raça Nelore (N), 43 vacas mestiças $\mathrm{CN}$, filhas de touros $\mathrm{C}(1 / 2 \mathrm{C} 1 / 2 \mathrm{Ne} 3 / 4 \mathrm{C}$ $1 / 4 \mathrm{~N})$ e 65 vacas mestiças NC, filhas de touros $\mathrm{N}$ (1/2 N 1/2 C; 3/4 N 1/4 C e 5/8 N 3/8 C). A idade das vacas variou de 3 a 12 anos, sendo agrupadas em quatro classes, primíparas ( 3 anos de idade), jovens (4-5 anos de idade), adultas (6-8 anos de idade) e velhas (+ 9 anos de idade). Os bezerros filhos de vacas mestiças $\mathrm{CN}$ foram obtidos pelo acasalamento destas com touros $\mathrm{N}$.
Os bezerros filhos de vacas mestiças NC foram obtidos pelo acasalamento destas com touros C.

Durante o período de aleitamento, vacas e bezerros foram mantidos em PN e submetidos a três manejos distintos: Manejo I - Sem suplementação, sendo os bezerros desmamados aos 63 dias; Manejo II Suplementação com farelo de arroz integral, equivalente a $0,7 \%$ do peso vivo das vacas, sendo os bezerros desmamados aos 42 dias de idade; Manejo III - Suplementação com farelo de arroz integral, equivalente a $0,7 \%$ do peso vivo das vacas, sendo os bezerros desmamados aos 63 dias de idade.

A lotação média foi de 0,9 vaca com cria ao pé/ha, sendo que a disponibilidade média de massa de forragem, medida por intermédio da técnica de dupla amostragem, durante o período de aleitamento foi de $4240 \mathrm{~kg}$ de MS/ha.

Os bezerros foram pesados nas primeiras 24 horas após o nascimento e, após, em intervalos de 21 dias até os 84 dias de idade, existindo variação de \pm 3 dias. Após, foram pesados aos 5, 7, 9 e 12 meses de idade, sendo que nesta ocasião foi atribuído a CC dos bezerros, seguindo uma escala de 1 a 5 ( 1 = muito magro, 2 = magro, 3 = médio, 4 = gordo e 5 = muito gordo).

Após o desmame, todos os bezerros foram submetidos ao mesmo manejo, recebendo a mesma alimentação. Nos primeiros cinco dias após o desmame permaneceram em curral, recebendo ração comercial contendo $18 \%$ de PB. Após, os bezerros permaneceram, em média, 30 dias em confinamento, onde receberam, como volumoso, silagem de milho e concentrado em quantidade equivalente a $1 \%$ do peso vivo, contendo $22 \%$ de PB, e constituído por farelo de soja (25\%), farelo de trigo (65\%), grão de sorgo triturado (5\%), calcário calcítico (4\%), sal (1\%), complexo mineral (120 g), o qual continha em sua fórmula 6,99; 6,90; 4,$01 ; 1,70 ; 0,18$ e $0,07 \%$, respectivamente, de Fe, $\mathrm{Zn}, \mathrm{Mn}, \mathrm{Cu}$, I e Co. Cada $100 \mathrm{~kg}$ de concentrado continha ionóforo (85 g de rumensin e $85 \mathrm{~g}$ de oxitetraciclina). Após este período, foram mantidos em pastagem de milheto (Penisetum americanum Leeke) recebendo o mesmo concentrado e na mesma quantidade até a primeira quinzena de março. A partir dos 5 meses até os 12 meses de idade, foram mantidos em PN, recebendo silagem de milho e concentrado contendo $18 \%$ de $\mathrm{PB}$, formulado a partir dos mesmos componentes do concentrado que receberam no confinamento. 
O delineamento experimental foi inteiramente casualizado em arranjo fatorial $4 \times 3$ (quatro grupos genéticos e três manejos), com número de repetições variável por tratamento. No manejo I, foram usados 10 bezerros $\mathrm{C}, 8$ bezerros $\mathrm{N}, 23$ bezerros mestiços com predominância de sangue $\mathrm{C}$, filhos de touros $\mathrm{C}$, 14 bezerros mestiços com predominância de sangue $\mathrm{N}$, filhos de touros $\mathrm{N}$ e no manejo II, 9 bezerros $\mathrm{C}$, 7 bezerros N, 19 bezerros mestiços com predominância de sangue $C$ filhos de touros C, 15 bezerros mestiços com predominância de sangue $\mathrm{N}$, filhos de touros $\mathrm{N}$, o manejo III era constituído por 10 bezerros C, 7 bezerros $N$, 23 bezerros mestiços com predominância de sangue $C$ filhos de touros $C, 14$ bezerros mestiços com predominância de sangue $\mathrm{N}$, filhos de touros $\mathrm{N}$.

Os dados coletados foram submetidos à análise de variância e, para as variáveis em que houve efeito significativo, as médias foram comparadas pelo teste Tukey, por intermédio do pacote estatístico SAS (1993).

Foi utilizado o seguinte modelo estatístico: $\mathrm{Y}_{\mathrm{ijklm}}=\mu+\mathrm{OP}_{\mathrm{i}}+\mathrm{M}_{\mathrm{j}}+\mathrm{GGV}_{\mathrm{k}}+\mathrm{IV}_{\mathrm{l}}+\mathrm{M}_{\mathrm{j}} \mathrm{GGV}_{\mathrm{k}}+\mathrm{M}_{\mathrm{j}}$ $* \mathrm{IV}_{1}+\mathrm{GGV}_{\mathrm{k}} * \mathrm{IV}_{1}+\mathrm{e}_{\mathrm{ijklm}}$, em que: $\mathrm{Y}_{\mathrm{ijkl}}=$ variáveis dependentes; $\mu=$ média geral de todas as observações; $\mathrm{OP}_{\mathrm{i}}=$ efeito da covariável ordem de nascimento de bezerro de ordem " $\mathrm{i}$ ”; $\mathrm{M}_{\mathrm{j}}$ = efeito do manejo de ordem “j”, sendo 1 = vacas não suplementadas e que desmamaram aos 63 dias, 2 = vacas suplementadas e que desmamaram aos 63 dias e $3=$ vacas suplementadas e que desmamaram aos 42 dias; $\mathrm{GGV}_{\mathrm{k}}$ = efeito do grupo genético da vaca de ordem " $k$ ", sendo $1=$ Charolês $(\mathrm{C}), 2=\operatorname{Nelore}(\mathrm{N}), 3=$ mestiças CN filhas de touros C e 4 = Mestiças NC filhas de touros $\mathrm{N} ; \mathrm{IV}_{1}=$ efeito da classe de idade da vaca de ordem "l", sendo 1 = primíparas, 2 = jovens, 3 = adultas e 4 = velhas; $\left(M^{*} G G V\right) i k=$ interação entre j-ésimo manejo e k-ésimo grupo genético; $\left(\mathrm{M}^{*} \mathrm{IV}\right) \mathrm{jl}$ = interação entre o j-ésimo manejo e lésimo classe de idade da vaca; $(\mathrm{GGV} * \mathrm{IV}) \mathrm{kl}=$ interação entre o k-ésimo grupo genético da vaca e lésimo classe de idade da vaca; $\mathrm{e}_{\mathrm{ijkl}}=$ erro associado a cada observação.

Para a variável dependente peso do bezerro ao nascer, o efeito de tipo de manejo foi retirado do modelo.

\section{Resultados e Discussão}

As variáveis avaliadas no presente estudo não foram afetadas pelo sexo do bezerro. Na Tabela 1, são apresentados os ganhos de peso diários dos bezerros do nascimento aos 12 meses de idade, de acordo com o manejo alimentar das vacas durante o aleitamento. Do nascimento aos 21 dias, o ganho de peso foi similar entre os diferentes manejos. Observa-se que, com o avanço do período de lactação, houve queda no GMD dos bezerros em todos os manejos. Considerando apenas os bezerros desmamados aos 63 dias, constatou-se decréscimo no GMD do nascimento aos 21 para o GMD dos 21 aos 42 dias de $22,4 \%$ para aqueles cujas vacas não foram suplementadas contra $7,3 \%$ para aqueles cujas vacas foram suplementadas, e dos 21 aos 42 para o GMD dos 42 aos 63 dias o decréscimo foi de 14,9 e 13,8\%, citados na mesma ordem, ou seja, a desaceleração no GMD foi maior para os bezerros filhos de vacas não suplementadas, principalmente até os 42 dias de idade. Esta queda foi conseqüência do declínio na produção de leite das vacas, pois, segundo Cerdótes et al. (2004), a produção de leite das mães destes bezerros caiu do primeiro para o segundo período (23,5 e $14,3 \%$, respectivamente, para as não suplementadas e suplementadas) e do segundo para o terceiro período a queda foi de 19,5 e 10,3\%, citados na mesma ordem. Estes resultados mostram a estreita relação entre a produção de leite da vaca com o ganho de peso do bezerro, particularmente na fase inicial da vida, onde o leite é a principal fonte de nutrientes. Concordando com os dados obtidos por Albuquerque et al. (1993), os quais verificaram alta correlação entre a produção de leite das vacas e o ganho de peso dos bezerros.

Considerando apenas os bezerros desmamados aos 63 dias, verifica-se, na Tabela 1, que bezerros filhos de vacas suplementadas apresentaram maior GMD do nascimento aos 63 dias (561 contra 457 g), ou seja, superioridade de 22,8\%. Assim como o GMD, a produção de leite das mães destes bezerros também foi superior em 34,1\% neste período (Cerdótes et al., 2004), comprovando a relação existente entre a produção de leite da vaca e o desempenho do bezerro. Através da diferença no GMD destes dois grupos de bezerros, pode-se inferir que a produção de leite das vacas não suplementadas não foi suficiente para suprir a demanda nutricional de seus bezerros, pois estes não conseguiram expressar seu potencial genético concordando com os dados obtidos por Moojen et al. (1994), os quais observaram maior GMD no período de aleitamento nos bezerros mantidos em melhor condição alimentar neste período. 
Outros autores também observaram maior GMD em bezerros filhos de vacas submetidas a melhores condições nutricionais no período de aleitamento. Em trabalho realizado por Restle et al. (2004), em que testaram o desempenho de bezerro ao pé da mãe, foi verificado que, até os 70 dias de idade, os bezerros mantidos em pastagem cultivada tiveram ganho de peso de $657 \mathrm{~g} / \mathrm{dia}$, ao passo que aqueles mantidos em PN apresentaram ganho de $485 \mathrm{~g} /$ dia. Segundo Neville Jr. (1962), quando o nível alimentar é melhor, maiores são a produção de leite da vaca e o ganho de peso do bezerro, concordando com os dados obtidos por Senna (1996), que verificou maior GMD e peso ao desmame em bezerros filhos de vacas mantidas em pastagem cultivada de melhor qualidade, quando comparados àqueles filhos de vacas mantidas exclusivamente em PN.

Observa-se, na Tabela 1, que nos bezerros desmamados aos 42 dias o GMD dos 42 aos 63 (360 g) caiu em 36,1\%, em relação ao período anterior (563 g)

Tabela 1 - Médias ajustadas e erros-padrão, em gramas, para o ganho de peso médio diário, do nascimento aos 21 dias (GMDnasc-21d), dos 21 aos 42 dias (GMD21-42d), dos 42 aos 63 dias (GMD42-63d), dos 63 aos 84 dias (GMD63-84), dos 84 dias aos 5 meses (GMD84d-5m), dos 5 aos 7 meses (GMD5-7m), dos 7 aos 9 meses (GMD7-9m), dos 9 aos 12 meses (GMD9-12m), dos 84 dias aos 12 meses (GMD84d-12m), dos 5 aos 12 meses (GMD5-12m), do nascimento aos 42 dias (GMDnasc-42d) e do nascimento aos 63 dias (GMDnasc-63d), de acordo com o manejo alimentar das vacas

Table 1 - Adjusted means and standard errors, in grams, for average daily weight gain, from birth to 21 days (ADGbirth-21d), from 21 to 42 days (ADG21-42d), from 42 to the 63 days (ADG42-63d), from 63 to 84 days (ADG63-84d), from 84 days to 5 months ( $A G D 84 d-5 m$ ), from 5 to 7 months (ADG5-7m), from 7 to 9 months (ADG7-9m), from 9 to 12 months (ADG9-12m), from 84 days to 12 months (ADG84d-12m), from 5 to 12 months (ADG5-12m), from birth to 42 days (ADGbirth-42d) and from birth to 63 days (ADGbirth-63d) of age, according to cow feeding management

\begin{tabular}{|c|c|c|c|}
\hline \multirow[b]{2}{*}{$\begin{array}{l}\text { Variáveis } \\
\text { Variables }\end{array}$} & \multicolumn{3}{|c|}{$\begin{array}{l}\text { Manejo alimentar das vacas } \\
\text { Cow feeding management }\end{array}$} \\
\hline & $\begin{array}{c}\text { Vacas não } \\
\text { suplementadas que } \\
\text { desmamaram aos } \\
63 \text { dias } \\
\text { Non supplemented } \\
\text { cows weaned at } 63 \text { days }\end{array}$ & $\begin{array}{c}\text { Vacas } \\
\text { suplementadas } \\
\text { que desmamaram } \\
\text { aos } 63 \text { dias } \\
\text { Supplemented cows } \\
\text { weaned at } 63 \text { days }\end{array}$ & $\begin{array}{c}\text { Vacas } \\
\text { suplementadas } \\
\text { que desmamaram } \\
\text { aos } 42 \text { dias } \\
\text { Supplemented cows } \\
\text { weaned at } 42 \text { days }\end{array}$ \\
\hline $\begin{array}{l}\text { GMDnasc-21d } \\
\text { ADGbirth-21d }\end{array}$ & $562 \pm 39 a$ & $617 \pm 43 a$ & $647 \pm 42 a$ \\
\hline $\begin{array}{l}\text { GMD21-42d } \\
A D G 21-42 d\end{array}$ & $436 \pm 34 b$ & $572 \pm 37 \mathrm{ab}$ & $563 \pm 36 a$ \\
\hline $\begin{array}{l}\text { GMD42-63d } \\
A D G 42-63 d\end{array}$ & $371 \pm 31 b$ & $493 \pm 34 a$ & $360 \pm 33 b$ \\
\hline $\begin{array}{l}\text { GMD63-84d } \\
\text { ADG63-84d }\end{array}$ & $438 \pm 46 a$ & $432 \pm 49 a$ & $352 \pm 47 a$ \\
\hline $\begin{array}{l}\text { GMD84d-5m } \\
\text { ADG84d-5m }\end{array}$ & $736 \pm 68 a$ & $775 \pm 73 a$ & $706 \pm 70 a$ \\
\hline $\begin{array}{l}\text { GMD5-7m } \\
\text { ADG5-7m }\end{array}$ & $426 \pm 25 a$ & $418 \pm 27 a$ & $357 \pm 26 a$ \\
\hline $\begin{array}{l}\text { GMD7-9m } \\
A D G 7-9 m\end{array}$ & $129 \pm 21 a$ & $101 \pm 23 a$ & $122 \pm 22 \mathrm{a}$ \\
\hline $\begin{array}{l}\text { GMD9-12m } \\
\text { ADG9-12 }\end{array}$ & $538 \pm 43 a$ & $531 \pm 98 a$ & $442 \pm 45 a$ \\
\hline $\begin{array}{l}\text { GMD84d-12m } \\
\text { ADG84d-12m }\end{array}$ & $435 \pm 19 a$ & $427 \pm 21 a$ & $386 \pm 20 a$ \\
\hline $\begin{array}{l}\text { GMD5-12m } \\
\text { ADG5-12m }\end{array}$ & $392 \pm 20 a$ & $380 \pm 22 a$ & $327 \pm 21 a$ \\
\hline $\begin{array}{l}\text { GMDnasc-42d } \\
\text { ADGbirth-42d }\end{array}$ & $499 \pm 24 b$ & $595 \pm 27 a$ & $603 \pm 26 a$ \\
\hline $\begin{array}{l}\text { GMDnasc-63d } \\
\text { ADGbirth-63d }\end{array}$ & $457 \pm 21 b$ & $561 \pm 23 a$ & $522 \pm 22 a$ \\
\hline
\end{tabular}

Médias na linha, seguidas por letras diferentes, diferem $(P<0,05)$ pelo teste Tukey.

Means, within a row, followed different letters, differ $(P<.05)$ by Tukey test. 
e continuou a declinar dos 63 aos 84 dias (352 g). Esta queda no desempenho mostra que estes animais tinham limitação do desenvolvimento do rúmen, pois, após a mudança na dieta alimentar, mesmo com uma fração considerável de concentrado, houve queda acentuada no desempenho. Além disso, deve ser considerado que a convivência do bezerro com a vaca foi interrompida com o desmame, causando estresse, o que também deve ter influenciado o desempenho. Já nos bezerros cujas vacas também foram suplementadas até os 63 dias, a queda no GMD no período imediatamente após o desmame foi de $12,4 \%$, bem inferior ao verificado nos bezerros desmamados aos 42 dias. Isto mostra que a amamentação ao pé da vaca por mais 21 dias foi muito importante. Provavelmente, este comportamento foi o resultado do melhor desenvolvimento do sistema digestivo dos bezerros. Os resultados do presente estudo estão de acordo com as observações de Story et al. (2000), que avaliaram o desempenho de bezerros desmamados em três idades distintas, 5, 7 ou 9 meses, observando maior GMD nos primeiros 28 dias pós-desmame em bezerros desmamados mais tardiamente, indicando que, até determinado momento, quanto mais velhos os bezerros ao desmame, menor é o estresse sofrido no período subsequente.

Já os bezerros desmamados aos 63 dias cujas vacas não foram suplementadas apresentaram incremento de $18,1 \%$ no GMD, no período imediatamente após o desmame, sendo numericamente o maior GMD neste período, $438 \mathrm{~g}$. Este comportamento no GMD indica que o rúmen estava melhor desenvolvido que nos bezerros dos outros manejos, provavelmente em função da necessidade de consumir pasto mais cedo que os demais, para atender às exigências de mantença e crescimento, uma vez que as mães produziam menos leite (Cerdótes et al., 2004). Segundo Lyford Jr. (1993), os ruminantes nascidos em meio ambiente natural têm acesso ao consumo de vegetais desde o nascimento, começando a pastar a partir da primeira ou segunda semana. Segundo o mesmo autor, o consumo de pasto promove rapidamente o desenvolvimento do rúmen tanto em tamanho como em funcionamento. A dieta fornecida aos bezerros logo após o desmame supriu melhor as necessidades destes, uma vez que a produção de leite das vacas e a qualidade do pasto limitavam o seu desempenho.

Analisando os dados obtidos no presente estudo e aqueles encontrados na literatura, pode-se afirmar que, quando há restrição alimentar severa para as vacas no período pós-parto, a produção de leite diminui e, por conseqüência, o desempenho dos bezerros piora. Nestas situações, desde que existam condições de ofertar ao bezerro uma dieta adequada, é mais aconselhável desmamar o bezerro mais precocemente, pois seu desempenho pode ser superior ao de bezerros ao pé da vaca.

Dos 84 dias aos 12 meses de idade, o GMD foi similar entre os três manejos. No entanto, observa-se que os filhos de vacas suplementadas desmamados aos 42 dias, na maioria dos períodos apresentaram GMD numericamente inferior ao GMD dos bezerros dos demais manejos, indicando que o desmame aos 42 dias teve conseqüência negativa sobre o desempenho subseqüente dos bezerros.

Na Tabela 2, encontram-se as médias de peso dos bezerros do nascimento aos 12 meses de idade, de acordo com o manejo alimentar das vacas no pósparto. O peso dos bezerros nos diferentes períodos foi reflexo direto do GMD.

Com relação aos bezerros desmamados aos 63 dias, observa-se que os filhos de vacas não suplementadas obtiveram GMD numericamente inferior dos 21 aos 42 dias, refletindo-se em menor peso aos 42 dias de idade e ainda foram mais leves ao desmame (59,9 contra 67,0 kg), indicando que o suplemento fornecido à vaca no período logo após o parto tem influência direta no desenvolvimento dos bezerros, em função da maior produção e qualidade do leite produzido por estas vacas (Cerdótes et al., 2004). Concordando com Albuquerque et al. (1993), os quais verificaram correlação alta e positiva entre a produção de leite da mãe e o peso dos bezerros, indicando que as vacas de maior produção de leite produzem bezerros mais pesados. Moojen et al. (1994), também verificaram maior peso ao desmame aos 101 dias de idade, em bezerros filhos de vacas mantidas em pastagem cultivada do que aqueles filhos de vacas mantidas em PN. Outros autores também verificaram melhor desempenho de bezerros filhos de vacas submetidas a melhor nível nutricional no período de lactação. Houghton et al. (1990), trabalhando com nível baixo ou alto de energia no pós-parto, verificaram que vacas que receberam dieta com nível alto de energia produziram bezerros mais pesados aos 60 dias de idade (100,8 contra 115,1 kg). Já Restle et al. (2004) relataram maior peso aos 70 dias de idade para bezerros que permaneceram juntamente com as vacas em pastagem cultivada $(79,3 \mathrm{~kg})$ em relação aos mantidos em PN (67,5 kg).

\section{R. Bras. Zootec., v.33, n.3, p.597-609, 2004}


Observa-se que a diferença no peso entre os bezerros desmamados aos 63 dias se manteve superior, até os 84 dias, naqueles cujas vacas foram suplementadas. Após os 84 dias, a diferença entre os dois grupos deixou de ser significativa. Estes resultados indicam que, embora a suplementação tenha favorecido o peso ao desmame e aos 84 dias, não há vantagem da sua adoção já que a diferença desaparece nos períodos subseqüentes, sendo o peso aos 12 meses praticamente igual aqueles de vacas não suplementadas. Constatação similar foi feita por Restle et al. (1999), os quais verificaram que o ganho de peso de bezerros desmamados aos três meses foi mais baixo até os sete meses do que os mantidos ao pé da vaca até esta idade. Devido ao maior ganho de peso após os sete meses, os bezerros desmamados precocemente não diferiram dos desmamados aos sete meses no peso aos 12, 18 e 24 meses de idade.

Em relação às diferentes idades de desmame, 42 ou 63 dias, dos bezerros filhos de vacas suplementadas, observa-se que os bezerros desmamados aos 42 dias apresentaram pesos similares até os 9 meses de idade, sendo inferiores aos 12 meses (189,4 contra 174,4 kg), o que foi reflexo do GMD destes até um ano de idade, pois, embora não tenha apresentado diferença estatística, foi numericamente inferior aos demais grupos de bezerros nestes períodos. $\mathrm{O}$ menor peso aos 12 meses dos bezerros desmamados aos 42 dias indica que não é aconselhável o desmame de bezerros em idade tão precoce. Segundo Simeone et al. (1997), em bezerros desmamados aos 78 dias de idade, o GMD até os 141 dias de idade foi de $243 \mathrm{~g}$ contra $590 \mathrm{~g}$ para os que ficaram ao pé da vaca até os 141 dias, quando foram desmamados. Esses autores verificaram que aos 15 meses de idade os animais desmamados aos 78 dias apresentavam peso significativamente inferior ao dos desmamados aos 141 dias (233,1 contra 249,6 kg). Esses resultados, aliados aos do presente estudo, sugerem que o baixo ganho de peso dos bezerros logo após o desmame tem conseqüência negativa no desempenho futuro dos bezerros.

Tabela 2 - Médias ajustadas e erros-padrão, em kg, para o peso dos bezerros ao nascimento (Pnasc), 21 (P21d), 42 (P42d), 63 (P63d) e 84 dias (P84d), aos 5 (P5m), 7 (P7m), 9 (P9m) e 12 meses (P12m) de idade, de acordo com o manejo alimentar das vacas

Table 2 - Adjusted means and standard errors, in kg, for calves weight at birth (Wbirth.), 21 (W21d), 42 (W42d), 63 (W63d) and 84 days (W84d), at 5 (W5m), 7 (W7m), 9 (W9m) and 12 months (W12m), according to cow feeding management

\begin{tabular}{|c|c|c|c|}
\hline \multirow[b]{2}{*}{$\begin{array}{l}\text { Variáveis } \\
\text { Variables }\end{array}$} & \multicolumn{3}{|c|}{$\begin{array}{l}\text { Manejo alimentar das vacas } \\
\text { Cow feeding management }\end{array}$} \\
\hline & $\begin{array}{c}\text { Vacas não } \\
\text { suplementadas que } \\
\text { desmamaram aos } \\
63 \text { dias } \\
\text { Non supplemented } \\
\text { cows weaned at } 63 \text { days }\end{array}$ & $\begin{array}{c}\text { Vacas } \\
\text { suplementadas } \\
\text { que desmamaram } \\
\text { aos } 63 \text { dias } \\
\text { Supplemented cows } \\
\text { weaned at } 63 \text { days }\end{array}$ & $\begin{array}{c}\text { Vacas } \\
\text { suplementadas } \\
\text { que desmamaram } \\
\text { aos } 42 \text { dias } \\
\text { Supplemented cows } \\
\text { weaned at } 42 \text { days }\end{array}$ \\
\hline $\begin{array}{l}\text { Pnasc. } \\
\text { Wbirth }\end{array}$ & $31,1 \pm 0,8 a$ & $31,7 \pm 0,8 a$ & $32,2 \pm 0,8 a$ \\
\hline $\begin{array}{l}\text { P21d } \\
\text { W21d }\end{array}$ & $42,9 \pm 1,8 \mathrm{a}$ & $44,6 \pm 1,1 \mathrm{a}$ & $45,7 \pm 1,1 \mathrm{a}$ \\
\hline $\begin{array}{l}\text { P42d } \\
W 42 d\end{array}$ & $52,1 \pm 1,2 b$ & $56,6 \pm 1,3 a$ & $57,5 \pm 1,2 \mathrm{a}$ \\
\hline $\begin{array}{l}\text { P63d } \\
\text { W63d }\end{array}$ & $59,9 \pm 1,4 b$ & $67,0 \pm 1,5 a$ & $65,0 \pm 1,5 \mathrm{ab}$ \\
\hline $\begin{array}{l}\text { P84d } \\
\text { W84d }\end{array}$ & $69,2 \pm 1,8 b$ & $76,0 \pm 1,9 a$ & $72,4 \pm 1,9 a b$ \\
\hline $\begin{array}{l}\mathrm{P} 5 \mathrm{~m} \\
W 5 \mathrm{~m}\end{array}$ & $96,3 \pm 2,3 a$ & $103,5 \pm 2,4 a$ & $100,4 \pm 2,3 a$ \\
\hline $\begin{array}{l}\text { P7m } \\
W 7 m\end{array}$ & $128,3 \pm 3,0 \mathrm{a}$ & $134,8 \pm 3,2 a$ & $127,2 \pm 3,1 \mathrm{a}$ \\
\hline $\begin{array}{l}\mathrm{P} 9 \mathrm{~m} \\
W 9 \mathrm{~m}\end{array}$ & $136,0 \pm 3,2 a$ & $141,1 \pm 3,5 a$ & $134,5 \pm 3,3 a$ \\
\hline $\begin{array}{l}\mathrm{P} 12 \mathrm{~m} \\
W 12 \mathrm{~m}\end{array}$ & $184,8 \pm 5,3 a b$ & $189,4 \pm 5,8 a$ & $174,4 \pm 5,5 b$ \\
\hline
\end{tabular}

Médias na linha, seguidas por letras diferentes, diferem $(P<0,05)$ pelo teste Tukey.

Means, within a row, followed by different letters, differ $(P<.05)$ by Tukey test. 
Na Tabela 3, encontram-se as médias para a CC dos bezerros aos 5, 7 e 12 meses de idade, de acordo com o manejo alimentar das vacas no pós-parto. Observa-se que os bezerros apresentaram CC semelhante nas diferentes idades. Na escala de 1-5 pontos, a CC dos bezerros ficou entre 2,5 e 3,0 pontos, ou seja, entre magra e média. Isto se deve ao fato de os bezerros estarem em fase de crescimento, e a dieta fornecida ter sido suficiente para promover ganhos muito altos, o que proporcionaria deposição de gordura, refletindo-se em CC mais elevada.

Na Tabela 4, encontram-se os valores médios para o GMD do nascimento aos 12 meses de idade dos bezerros, de acordo com o grupo genético da mãe. Verifica-se que o GMD do nascimento ao desmame foi maior para os bezerros mestiços em relação aos $\mathrm{C}$, sendo que os bezerros $\mathrm{N}$ apresentaram GMD intermediário. O maior GMD neste período para os bezerros mestiços foi reflexo direto da produção de leite de suas mães, pois segundo Cerdótes et al. (2004), vacas mestiças produziram mais leite, seguidas das vacas $\mathrm{N}$ e $\mathrm{C}$, respectivamente, constatando-se, conforme citado anteriormente, que o GMD é conseqüência direta da produção de leite da vaca na fase inicial da vida dos bezerros (Neville Jr., 1962; Rutledge et al., 1971; Robinson et al., 1978; Senna, 1996; Quadros \& Lobato, 1997). Além da produção de leite, em função da heterozigose das vacas, o melhor desempenho dos bezerros mestiços também está associado à heterozigose individual (Restle et al.,
1999). Alencar (1989), avaliando o desempenho de bezerros das raças Canchim e $\mathrm{N}$, observou maior produção de leite nas vacas Canchim aos 30 e 60 dias e também tendência de os bezerros Canchim apresentarem maior GMD do nascimento aos 60 dias, em relação aos bezerros $\mathrm{N}$.

Ainda na Tabela 4, observa-se que, no período logo após o desmame, os bezerros de todos grupos apresentaram declínio no GMD. Bezerros N foram os que apresentaram menor GMD (219 g) logo após o desmame, sendo que os demais grupos não diferiram entre si. A queda acentuada no GMD após o desmame dos bezerros $\mathrm{N}$ desmamados aos 42 ou 63 dias indica que ainda não possuíam o rúmen totalmente desenvolvido e, por isso, sofreram mais com a falta de leite em sua dieta. Outra explicação para esta queda pode estar associada à ausência da mãe, talvez a relação bezerro/vaca seja mais forte no $\mathrm{N}$ do que nos demais grupos genéticos.

Dos 84 dias aos 12 meses de idade, o GMD continuou inferior nos bezerros N. Alencar (1989) também verificou menor GMD em bezerros $\mathrm{N}$ do nascimento até os 7 meses, quando comparados a bezerros Canchim.

Encontram-se na Tabela 5 as médias para o peso dos bezerros do nascimento aos 12 meses de idade, de acordo com o grupo genético da vaca. O menor peso ao nascimento, verificado nos bezerros $\mathrm{N}$ em relação aos demais grupos genéticos está de acordo com a média do peso ao nascer para esta raça, sendo

Tabela 3 - Médias ajustadas e erros-padrão, em pontos, para a condição corporal dos bezerros aos 5 $(\mathrm{CC} 5 \mathrm{~m}), 7(\mathrm{CC} 7 \mathrm{~m})$ e 12 meses $(\mathrm{CC} 12 \mathrm{~m})$ de idade, de acordo com o manejo alimentar das vacas Table 3 - Adjusted means and standard errors, in points, for body condition of calves at 5 (BC5m), 7 (BC7m) and 12 months (BC12m) of age, according to cow feeding management

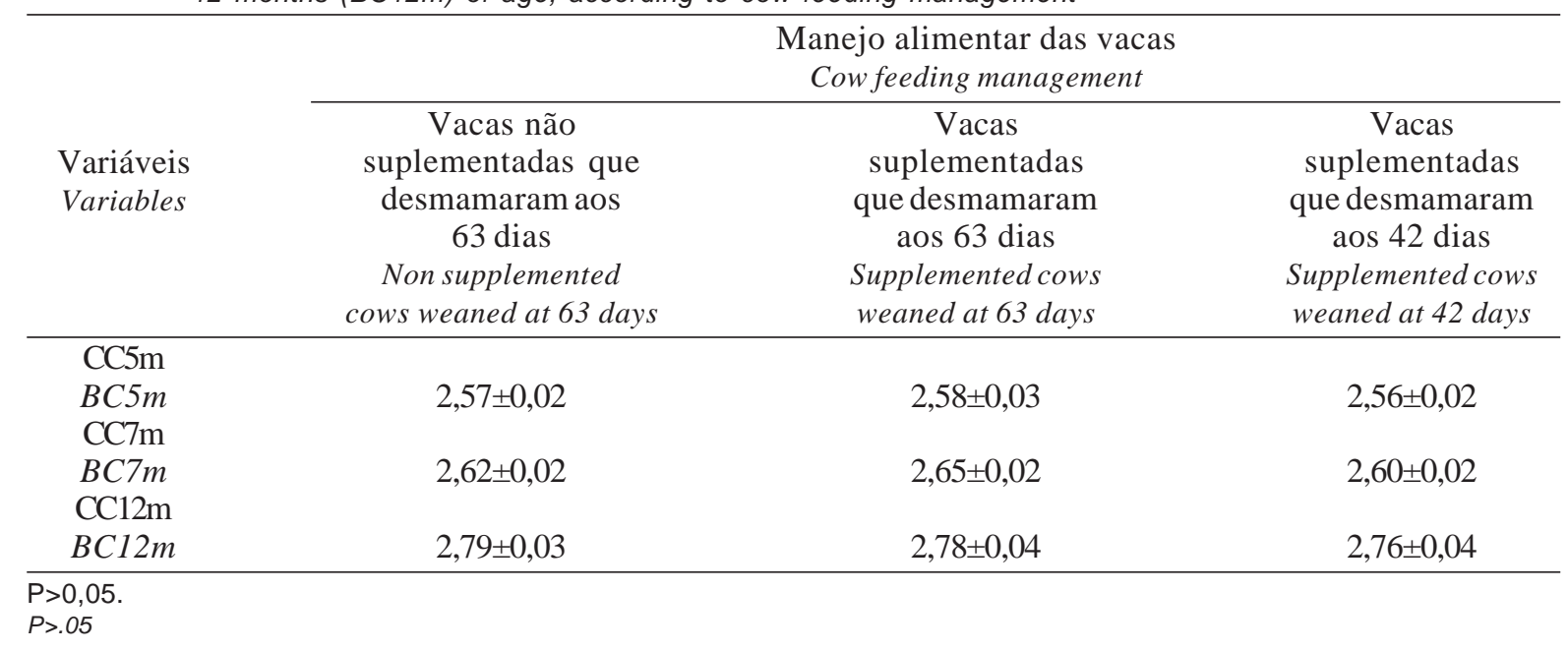

R. Bras. Zootec., v.33, n.3, p.597-609, 2004 
que Albuquerque et al. (1993) verificaram peso ao nascimento de bezerros $\mathrm{N}(28,5 \mathrm{~kg})$, ou seja, similar ao peso verificado no presente estudo (27,8 kg).

Verifica-se ainda na Tabela 5 que os bezerros $\mathrm{N}$ apresentaram menor peso em relação aos mestiços, em todos os períodos avaliados. Comparando os bezerros puros entre si, verifica-se que os $\mathrm{N}$ apresentaram menor peso na maioria dos períodos avaliados. No entanto, ao desmame e aos 84 dias de idade apresentaram peso similar. A similaridade do peso ao desmame nos bezerros puros se deve à melhor produção de leite das vacas $\mathrm{N}$ em relação às vacas $\mathrm{C}$ durante a lactação (Cerdótes et al., 2004), o que proporcionou ganho de peso numericamente superior para os bezerros $\mathrm{N}$ no pré-desmame, compensando, dessa forma, o menor peso observado ao nascimento para estes bezerros. Já o GMD do desmame aos 84 dias foi inferior para os bezerros N (Tabela 4), porém, como foi apenas durante breve período, não chegou a refletir em menor peso aos 84 dias.

A partir dos 84 dias de idade, o GMD dos bezerros $\mathrm{C}$ foi similar ou superior ao GMD dos bezerros N, o que resultou em menor peso dos bezerros $\mathrm{N}$ em relação aos $\mathrm{C}$ a partir do 5 o mês de idade.

Tabela 4 - Médias ajustadas e erros-padrão, em gramas, para o ganho de peso médio diário dos bezerros, do nascimento aos 21 dias (GMDnasc-21d), dos 21 aos 42 dias (GMD21-42d), dos 42 aos 63 dias (GMD42-63d), do nascimento ao desmame (GMDnasc-desm), do desmame aos 84 dias (GMDdesm-84d), dos 84 aos 5 meses (GMD84d-5m), dos 5 aos 7 meses (GMD5-7m), dos 7 aos 9 meses (GMD7-9m), dos 9 aos12 meses (GMD9-12m), dos 84 dias aos 12 meses (GMD84d-12m) e dos 5 aos 12 meses de idade, de acordo com o grupo genético das vacas

Table 4 - Adjusted means and standard errors, in grams, for average daily weight gain, from birth to 21 days (ADGbirth-21d), from 21 to 42 days (ADG21-42d), from 42 to 63 days (ADG42-63d), from birth to weaning (ADGbirth-weaning), from weaning to 84 days (ADGweaning-84d), from 84 days to 5 months (AGD84d-5m), from 5 to 7 months (ADG5-7m), from 7 to 9 months (ADG7-9m), from 9 to 12 months (ADG9-12m), from 84 days to 12 months (ADG84d-12m) and from 5 to 12 months (ADG5-12m) of age, according to cow genetic group

\begin{tabular}{|c|c|c|c|c|}
\hline \multirow[t]{2}{*}{$\begin{array}{l}\text { Variáveis } \\
\text { Variables }\end{array}$} & \multicolumn{4}{|c|}{$\begin{array}{l}\text { Grupo genético da vaca } \\
\text { Cow genetic group }\end{array}$} \\
\hline & $\begin{array}{l}\text { Charolês (C) } \\
\text { Charolais (C) }\end{array}$ & $\begin{array}{l}\text { Nelore }(\mathrm{N}) \\
\text { Nellore }(N)\end{array}$ & $\begin{array}{l}\text { Mestiças CN } \\
\text { CN crossbreds }\end{array}$ & $\begin{array}{l}\text { Mestiças NC } \\
\text { NC crossbreds }\end{array}$ \\
\hline $\begin{array}{l}\text { GMDnasc-21d } \\
\text { ADGbirth-21d }\end{array}$ & $555 \pm 54 a$ & $549 \pm 58 a$ & $670 \pm 44 a$ & $661 \pm 34 a$ \\
\hline $\begin{array}{l}\text { GMD21-42d } \\
A D G 21-42 d\end{array}$ & $429 \pm 47 \mathrm{~b}$ & $574 \pm 50 \mathrm{a}$ & $570 \pm 38 a$ & $520 \pm 29 \mathrm{ab}$ \\
\hline $\begin{array}{l}\text { GMD42-63d } \\
A D G 42-63 d\end{array}$ & $281 \pm 43 b$ & $444 \pm 46 a$ & $409 \pm 35 a b$ & $498 \pm 27 a$ \\
\hline $\begin{array}{l}\text { GMDnasc.-desm } \\
\text { ADGbirth-weaning }\end{array}$ & $440 \pm 30 b$ & $558 \pm 32 a b$ & $592 \pm 24 a$ & $570 \pm 19 a$ \\
\hline $\begin{array}{l}\text { GMDdesm-84d } \\
\text { ADGweaning-84d }\end{array}$ & $426 \pm 62 a$ & $219 \pm 66 b$ & $426 \pm 51 \mathrm{a}$ & $558 \pm 39 a$ \\
\hline $\begin{array}{l}\text { GMD84d-5m } \\
\text { ADG84d-5m }\end{array}$ & $766 \pm 92 a$ & $620 \pm 99 a$ & $754 \pm 77 a$ & $818 \pm 58 a$ \\
\hline $\begin{array}{l}\text { GMD5-7m } \\
\text { AGD5-7m }\end{array}$ & $335 \pm 34 b$ & $360 \pm 37 b$ & $480 \pm 29 a$ & $427 \pm 22 \mathrm{ab}$ \\
\hline $\begin{array}{l}\text { GMD7-9m } \\
A D G 7-9 m\end{array}$ & $132 \pm 29 a$ & $40 \pm 31 b$ & $144 \pm 24 a b$ & $154 \pm 18 a$ \\
\hline $\begin{array}{l}\text { GMD9-12m } \\
A D G 9-12 m\end{array}$ & $673 \pm 59 a$ & $287 \pm 64 b$ & $452 \pm 49 a$ & $604 \pm 37 a$ \\
\hline $\begin{array}{l}\text { GMD84d-12m } \\
\text { ADG84d-12m }\end{array}$ & $460 \pm 26 a$ & $300 \pm 28 \mathrm{~b}$ & $426 \pm 21 \mathrm{a}$ & $478 \pm 16 a$ \\
\hline $\begin{array}{l}\text { GMD5-12m } \\
\text { ADG5-12m }\end{array}$ & $416 \pm 28 \mathrm{a}$ & $447 \pm 30 \mathrm{~b}$ & $377 \pm 23 a$ & $425 \pm 17 a$ \\
\hline
\end{tabular}

Médias na linha, seguidas por letras diferentes, diferem $(P<0,05)$ pelo teste Tukey.

Means, within a row, followed by different letters, differ $(P<.05)$ by Tukey test.

R. Bras. Zootec., v.33, n.3, p.597-609, 2004 
Tabela 5 - Médias ajustadas e erros-padrão, em $\mathrm{kg}$, para o peso dos bezerros ao nascimento (Pnasc), 21 (P21d), 42 (P42d), 63 (P63d), ao desmame (P desmame) e 84 dias (P84d) de idade, aos 5 (P5m), 7 (P7m), 9 (P9m) e 12 meses (P12m) de idade, de acordo com o grupo genético das vacas

Table 5 - Adjusted means and standard errors, in kg, for calves weight at birth (Wbirth.), 21 (W21d), 42 (W42d), 63 (W63d), at weaning (W weaning) and 84 (W84d) days of age, at 5 (W5m), 7 (W7m), 9 (W9m) and 12 months (W12m), according to cow genetic group

\begin{tabular}{|c|c|c|c|c|}
\hline \multirow[t]{2}{*}{$\begin{array}{l}\text { Variáveis } \\
\text { Variables }\end{array}$} & \multicolumn{4}{|c|}{$\begin{array}{c}\text { Grupo genético das vacas } \\
\text { Cow genetic group }\end{array}$} \\
\hline & $\begin{array}{l}\text { Charolês (C) } \\
\text { Charolais (C) }\end{array}$ & $\begin{array}{l}\text { Nelore }(\mathrm{N}) \\
\text { Nellore }(N)\end{array}$ & $\begin{array}{l}\text { Mestiças CN } \\
\text { CN crossbreds }\end{array}$ & $\begin{array}{l}\text { Mestiças NC } \\
\text { NC crossbreds }\end{array}$ \\
\hline $\begin{array}{l}\text { Pnasc } \\
\text { Wbirth }\end{array}$ & $32,8 \pm 1,1 \mathrm{a}$ & $27,8 \pm 1,1 b$ & $34,4 \pm 0,9 a$ & $31,5 \pm 0,7 \mathrm{a}$ \\
\hline $\begin{array}{l}\text { P21d } \\
W 21 d\end{array}$ & $44,4 \pm 1,4 b$ & $39,4 \pm 1,5 c$ & $48,5 \pm 1,1 \mathrm{a}$ & $45,4 \pm 0,9 a b$ \\
\hline $\begin{array}{l}\text { P42d } \\
\text { W42d }\end{array}$ & $53,4 \pm 1,6 \mathrm{bc}$ & 51,4×1,7c & $60,5 \pm 1,3 \mathrm{a}$ & $56,3 \pm 1,0 \mathrm{ab}$ \\
\hline $\begin{array}{l}\text { P63d } \\
\text { W63d }\end{array}$ & $59,3 \pm 1,9 b$ & $60,8 \pm 2,1 b$ & $69,1 \pm 1,6 a$ & $66,7 \pm 1,2 \mathrm{a}$ \\
\hline $\begin{array}{l}\text { Pdesmame } \\
\text { Wweaning }\end{array}$ & $57,3 \pm 1,8 b$ & $58,9 \pm 2,0 \mathrm{~b}$ & $66,7 \pm 1,5 a$ & $63,2 \pm 1,1 \mathrm{a}$ \\
\hline $\begin{array}{l}\text { P84d } \\
\text { W84d }\end{array}$ & $68,2 \pm 2,4 b$ & $65,3 \pm 2,6 b$ & $78,2 \pm 2,0 a$ & $78,4 \pm 1,5 a$ \\
\hline $\begin{array}{l}\text { P5m } \\
W 5 m\end{array}$ & $96,5 \pm 3,1 \mathrm{a}$ & $89,0 \pm 3,3 b$ & $105,2 \pm 2,6 a$ & $109,4 \pm 1,9 a$ \\
\hline $\begin{array}{l}\text { P7m } \\
W 7 m\end{array}$ & $121,7 \pm 4,1 b$ & $116,0 \pm 4,2 \mathrm{c}$ & $141,2 \pm 3,4 a$ & $141,4 \pm 2,5 a b$ \\
\hline $\begin{array}{l}\mathrm{P} 9 \mathrm{~m} \\
W 9 m\end{array}$ & $129,6 \pm 4,3 b$ & $118,5 \pm 4,7$ c & $150,1 \pm 3,6 a$ & $150,7 \pm 2,7 a b$ \\
\hline $\begin{array}{l}\mathrm{P} 12 \mathrm{~m} \\
W 12 \mathrm{~m}\end{array}$ & $190,5 \pm 7,1 \mathrm{a}$ & $144,9 \pm 7,8 b$ & $190,8 \pm 6,0 a$ & $205,4 \pm 4,5 a$ \\
\hline
\end{tabular}

Médias na linha, seguidas por letras diferentes, diferem $(P<0,05)$ pelo teste Tukey.

Means, within a row, followed by different letters, differ $(P<.05)$ by Tukey test.

Tabela 6 - Médias ajustadas e erros-padrão, em pontos, para a condição corporal dos bezerros aos $5(\mathrm{CC} 5 \mathrm{~m}), 7(\mathrm{CC} 7 \mathrm{~m})$ e 12 meses $(\mathrm{CC} 12 \mathrm{~m})$ de idade, de acordo com o grupo genético das vacas

Table 6 - Adjusted means and standard errors, in points, for body condition of calves at 5 (BC5m), 7 (BC7m) and 12 months $(B C 12 m)$ of age, according to cow genetic group

\begin{tabular}{|c|c|c|c|c|}
\hline \multirow[t]{2}{*}{$\begin{array}{l}\text { Variáveis } \\
\text { Variables }\end{array}$} & \multicolumn{4}{|c|}{$\begin{array}{c}\text { Grupo genético das vacas } \\
\text { Cow genetic group }\end{array}$} \\
\hline & $\begin{array}{l}\text { Charolês (C) } \\
\text { Charolais (C) }\end{array}$ & $\begin{array}{l}\text { Nelore }(\mathrm{N}) \\
\text { Nellore }(N)\end{array}$ & $\begin{array}{l}\text { Mestiças CN } \\
\text { CN crossbreds }\end{array}$ & $\begin{array}{l}\text { Mestiças NC } \\
\text { NC crossbreds }\end{array}$ \\
\hline $\begin{array}{l}\text { CC5m } \\
\text { BC5m }\end{array}$ & $2,66 \pm 0,03 a$ & $2,41 \pm 0,03 b$ & $2,58 \pm 0,03 a$ & $2,62 \pm 0,02 a$ \\
\hline $\begin{array}{l}\mathrm{CC} 7 \mathrm{~m} \\
\mathrm{BC} 7 \mathrm{~m}\end{array}$ & $2,61 \pm 0,03 a$ & $2,53 \pm 0,03 b$ & $2,69 \pm 0,02 a$ & $2,66 \pm 0,02 a$ \\
\hline $\begin{array}{l}\mathrm{CC} 12 \mathrm{~m} \\
\text { BC12m }\end{array}$ & $2,98 \pm 0,05 a$ & $2,49 \pm 0,05 c$ & $2,73 \pm 0,04 b$ & 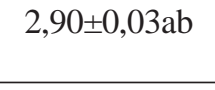 \\
\hline
\end{tabular}

Médias na linha, seguidas por letras diferentes, diferem $(P<0,05)$ pelo teste Tukey.

Means, within a row, followed by different letters, differ $(P<0.05)$ by Tukey test. 
Dos 9 aos 12 meses de idade, os bezerros C apresentaram peso mais elevado, principalmente em relação aos N (Tabela 4), atingindo peso aos 12 meses semelhante ao peso observado nos mestiços. Estes resultados estão de acordo com dados obtidos em anos anteriores em animais dos mesmos grupos genéticos, provenientes do mesmo rebanho experimental, no qual os bezerros $\mathrm{C}$ no período de inverno apresentaram melhor desempenho, quando comparados aos bezerros $\mathrm{N}$. Isso pode ser explicado pelo fato de bezerros desta raça serem mais adaptados ao frio e constituírem uma raça que, durante sua evolução, foi melhorada para alcançar maior ganho de peso e maior desenvolvimento muscular.

Bezerros mestiços CN e NC apresentaram peso semelhante, sendo mais pesados ao desmame do que os puros, não diferindo dos $C$ no peso aos 12 meses.

Na Tabela 6, encontram-se as médias para a CC dos bezerros dos 5 aos 12 meses de idade, de acordo com o grupo genético da vaca. Verifica-se que os bezerros $\mathrm{N}$ foram os que apresentaram pior CC em todos os períodos avaliados, sendo que os valores variaram de 2,41 a 2,53 pontos. Observa-se também que a CC aos 5, 7 e 12 meses de idade acompanhou a evolução do GMD dos bezerros, sendo maior para aqueles que apresentaram melhor GMD no período imediatamente anterior à pesagem dos bezerros, o que é lógico, já que a CC é a medida subjetiva do desenvolvimento muscular e deposição de gordura dos animais.

Aos 5 e aos 7 meses de idade, os bezerros mestiços foram os que apresentaram maior CC, porém sem diferir dos bezerros C. Já aos 12 meses, os bezerros $\mathrm{C}$ foram os que apresentaram maior CC, não diferindo dos NC, que, por sua vez, não diferiram dos CN, respectivamente de 2,98; 2,90 e 2,73 pontos.

Tabela 7 - Médias ajustadas e erros-padrão, em gramas, para o ganho de peso médio diário dos bezerros, do nascimento aos 21 dias (GMDnasc-21d), dos 21 aos 42 dias (GMD21-42d), dos 42 aos 63 dias (GMD42-63d), do nascimento ao desmame (GMDnasc-desm), do desmame aos 84 dias (GMDdesm-84d), dos 84 aos 5 meses (GMD84d-5m), dos 5 aos 7 meses (GMD5-7m), dos 7 aos 9 meses (GMD7-9m) e dos 9 aos12 meses (GMD9-12m) de idade, de acordo com a classe de idade das vacas

Table 7 - Adjusted means and standard errors, in grams, for average daily weight gain, from birth to 21 days (ADGbirth-21d), 21 to 42 days (ADG21-42d), 42 to 63 days (ADG 42-63d), from birth to weaning (ADGbirth-weaning), from weaning to 84 days (Wweaning-84d), from 84 days to 5 months (ADG84d-5m), from 5 to 7 (ADG 5-7m), 7 to 9 (ADG 7-9m) and 9 to 12 months (ADG 9$12 \mathrm{~m}$ ) of age, according to cows age class

\begin{tabular}{|c|c|c|c|c|}
\hline \multirow[t]{2}{*}{$\begin{array}{l}\text { Variáveis } \\
\text { Variables }\end{array}$} & \multicolumn{4}{|c|}{$\begin{array}{c}\text { Classe de idade das vacas } \\
\text { Cow age class }\end{array}$} \\
\hline & $\begin{array}{l}\text { Primíparas } \\
\text { First calf }\end{array}$ & $\begin{array}{c}\text { Jovens } \\
\text { Young }\end{array}$ & $\begin{array}{l}\text { Adultas } \\
\text { Adult }\end{array}$ & $\begin{array}{l}\text { Velhas } \\
\text { Old }\end{array}$ \\
\hline $\begin{array}{l}\text { GMDnasc-21d } \\
\text { ADGbirth-21d }\end{array}$ & $457 \pm 54 b$ & $657 \pm 43 a b$ & $681 \pm 37 \mathrm{ab}$ & $640 \pm 58 a$ \\
\hline $\begin{array}{l}\text { GMD21-42d } \\
A D G 21-42 d\end{array}$ & $439 \pm 46 b$ & $565 \pm 37 a$ & $517 \pm 32 \mathrm{ab}$ & $573 \pm 50 a$ \\
\hline $\begin{array}{l}\text { GMD42-63d } \\
\text { ADG42-63d }\end{array}$ & $283 \pm 43 b$ & 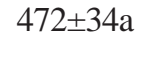 & $444 \pm 29 a$ & $432 \pm 47 a$ \\
\hline $\begin{array}{l}\text { GMDnasc-desm } \\
\text { ADGbirth-weaning }\end{array}$ & $411 \pm 30 b$ & $584 \pm 24 a$ & $583 \pm 20 a$ & 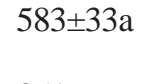 \\
\hline $\begin{array}{l}\text { GMDdesm-84d } \\
\text { ADGweaning-84d }\end{array}$ & $367 \pm 63 a$ & $464 \pm 49 a$ & $447 \pm 42 a$ & $351 \pm 67 a$ \\
\hline $\begin{array}{l}\text { GMD84-5m } \\
A D G 84-5 m\end{array}$ & $672 \pm 93 a$ & 691土73а & 770ะ63а & $825 \pm 101 a$ \\
\hline $\begin{array}{l}\text { GMD5-7m } \\
\text { ADG5-7m }\end{array}$ & $405 \pm 35 a$ & $387 \pm 27 a$ & $417 \pm 24 a$ & $393 \pm 38 a$ \\
\hline $\begin{array}{l}\text { GMD7-9m } \\
A D G 7-9 m\end{array}$ & $120 \pm 29 a$ & $122 \pm 23 a$ & $136 \pm 20 a$ & $92,0 \pm 32 a$ \\
\hline $\begin{array}{l}\text { GMD9-12m } \\
\text { ADG9-12m }\end{array}$ & $547 \pm 60 a$ & $471 \pm 46 a$ & $513 \pm 42 a$ & $484 \pm 64 a$ \\
\hline
\end{tabular}

Médias na linha, seguidas por letras diferentes, diferem $(P<0,05)$ pelo teste Tukey.

Means, within a row, followed by different letters, differ $(P<.05)$ by Tukey test.

R. Bras. Zootec., v.33, n.3, p.597-609, 2004 
Todos os bezerros apresentaram CC inferior a 3, indicando que estavam basicamente depositando tecido muscular.

Na Tabela 7, encontram-se as médias para o GMD dos bezerros do nascimento aos 12 meses de idade, de acordo com a classe de idade das vacas. Observa-se que do nascimento ao desmame os bezerros filhos de vacas prímiparas foram os que apresentaram menor GMD. Como o desenvolvimento dos bezerros no pré-parto está intimamente relacionado com a produção de leite de suas mães, o menor GMD observado nos filhos das prímiparas é reflexo da produção de leite destas, pois, segundo Cerdótes et al. (2004), estas vacas foram as que apresentaram menor produção leiteira. Segundo estes autores, a menor produção de leite dessa categoria de vacas é conseqüência do seu crescimento, pois, além dos nutrientes necessários para mantença e lactação, ainda necessitam de nutrientes para completar seu desenvolvimento.

Os resultados deste estudo concordam com os dados obtidos por Nelson et al. (1982), que, traba- lhando com diferentes grupos genéticos de vacas, com idade variando de dois a sete anos, verificaram que os bezerros filhos de vacas com idade superior a três anos, foram os que apresentaram maior GMD do nascimento aos 210 dias de idade, sendo que os filhos de vacas com três anos foram os que apresentaram desempenho intermediário e superior ao verificado nas vacas com dois anos de idade.

O melhor desempenho dos bezerros filhos de vacas jovens, adultas e velhas no pré-desmame é conseqüência das melhores condições fisiológicas desses animais em relação às prímiparas, resultando em maior produção de leite. Observa-se que, a partir do desmame, o GMD foi similar para todos os grupos de bezerros, indicando que a classe de idade da vaca influencia significativamente o GMD do bezerro somente no período pré-desmame.

Na Tabela 8, encontram-se as médias para o peso dos bezerros do nascimento aos 12 meses de idade, de acordo com a classe de idade da vaca. Observa-se que as prímiparas foram as que produziram bezerros mais

Tabela 8 - Médias ajustadas e erros-padrão, em $\mathrm{kg}$, para o peso dos bezerros ao nascimento (Pnasc), 21 (P21d), 42 (P42d), 63 (P63d), ao desmame (Pdesm) e aos 84 dias (P84d), aos 5 (P5m), 7 (P7m), 9 (P9m) e 12 meses (P12m) de idade, de acordo com a classe de idade das vacas

Table 8 - Adjusted means and standard errors, in $\mathrm{kg}$, for calves weight at birth (Wbirth), 21 (W21d), 42 (W42d), 63 (W63d) and 84 days of age (W84d), at 5 (W5m), 7 (W7m), 9 (W9m) and 12 (W12m) months of age, according to cow age class

\begin{tabular}{|c|c|c|c|c|}
\hline \multirow[t]{2}{*}{$\begin{array}{l}\text { Variáveis } \\
\text { Variables }\end{array}$} & \multicolumn{4}{|c|}{$\begin{array}{c}\text { Classe de idade das vacas } \\
\text { Cow age class }\end{array}$} \\
\hline & $\begin{array}{l}\text { Primíparas } \\
\text { First calf }\end{array}$ & $\begin{array}{l}\text { Jovens } \\
\text { Young }\end{array}$ & $\begin{array}{l}\text { Adultas } \\
\text { Adult }\end{array}$ & $\begin{array}{l}\text { Velhas } \\
\text { Old }\end{array}$ \\
\hline $\begin{array}{l}\text { Pnasc. } \\
\text { Wbirth }\end{array}$ & $29,2 \pm 1,1 b$ & $32,3 \pm 0,8 a$ & $32,6 \pm 0,7 \mathrm{a}$ & $32,5 \pm 1,1 \mathrm{ab}$ \\
\hline $\begin{array}{l}\text { P21d } \\
\text { W21d }\end{array}$ & $38,8 \pm 1,4 \mathrm{~b}$ & $46,0 \pm 1,1 \mathrm{a}$ & $46,9 \pm 1,0 \mathrm{a}$ & $46,0 \pm 1,5 a$ \\
\hline $\begin{array}{l}\text { P42d } \\
\text { W42d }\end{array}$ & $48,0 \pm 1,6 b$ & $57,8 \pm 1,3 \mathrm{a}$ & $57,8 \pm 1,1 \mathrm{a}$ & $58,0 \pm 1,7 \mathrm{a}$ \\
\hline $\begin{array}{l}\text { P63d } \\
\text { W63d }\end{array}$ & $50,0 \pm 2,0 \mathrm{~b}$ & $67,7 \pm 1,5 \mathrm{a}$ & $67,1 \pm 1,3 \mathrm{a}$ & $67,1 \pm 2,1 \mathrm{a}$ \\
\hline $\begin{array}{c}\text { Pdesm } \\
\text { Wweaning }\end{array}$ & $52,1 \pm 1,8 b$ & $64,7 \pm 1,4 \mathrm{a}$ & $64,6 \pm 1,3 \mathrm{a}$ & $64,8 \pm 2,0 \mathrm{a}$ \\
\hline $\begin{array}{l}\text { P84d } \\
\text { W84d }\end{array}$ & $61,8 \pm 2,5 b$ & $77,4 \pm 1,9 a$ & $76,5 \pm 1,7 \mathrm{a}$ & $74,4 \pm 2,7 \mathrm{a}$ \\
\hline $\begin{array}{l}\mathrm{P} 5 \mathrm{~m} \\
W 5 m\end{array}$ & $90,0 \pm 3,1 b$ & $104,2 \pm 2,4 a$ & $103,3 \pm 2,1 \mathrm{a}$ & $102,7 \pm 3,4 a$ \\
\hline $\begin{array}{l}\text { P7m } \\
W 7 m\end{array}$ & $120,4 \pm 4,1 b$ & $133,2 \pm 3,2 a$ & $134,6 \pm 2,8 a$ & $132,2 \pm 4,4 \mathrm{ab}$ \\
\hline $\begin{array}{l}\mathrm{P} 9 \mathrm{~m} \\
W 9 m\end{array}$ & $127,6 \pm 4,4 \mathrm{a}$ & $140,6 \pm 3,4 a$ & $143,0 \pm 3,0 a$ & $137,8 \pm 4,7 a$ \\
\hline $\begin{array}{l}\mathrm{P} 12 \mathrm{~m} \\
W 12 \mathrm{~m}\end{array}$ & $177,2 \pm 7,3 a$ & $184,3 \pm 5,6 a$ & $189,4 \pm 5,1 \mathrm{a}$ & $181,6 \pm 7,8 a$ \\
\hline
\end{tabular}

Médias na linha, seguidas por letras diferentes, diferem $(P<0,05)$ pelo teste Tukey.

Means, within a row, followed by different letters, differ $(P<.05)$ by Tukey test.

R. Bras. Zootec., v.33, n.3, p.597-609, 2004 
Tabela 9 - Médias ajustadas e erros-padrão, em pontos, para a condição corporal dos bezerros aos $5(\mathrm{CC} 5 \mathrm{~m}), 7(\mathrm{CC} 7 \mathrm{~m})$ e 12 meses $(\mathrm{CC} 12 \mathrm{~m})$ de idade, de acordo com a classe de idade das vacas

Table 9 - Adjusted means and standard errors, in points, for calves body condition at 5 (BC5m), 7 (BC7m) and 12 months (BC12m) of age, according to cow age class

\begin{tabular}{|c|c|c|c|c|}
\hline \multirow[t]{2}{*}{$\begin{array}{l}\text { Variáveis } \\
\text { Variables }\end{array}$} & \multicolumn{4}{|c|}{$\begin{array}{c}\text { Classes de idade das vacas } \\
\text { Cow age class }\end{array}$} \\
\hline & $\begin{array}{l}\text { Primíparas } \\
\text { First calf }\end{array}$ & $\begin{array}{l}\text { Jovens } \\
\text { Young }\end{array}$ & $\begin{array}{l}\text { Adultas } \\
\text { Adult }\end{array}$ & $\begin{array}{l}\text { Velhas } \\
\text { Old }\end{array}$ \\
\hline $\begin{array}{l}\text { CC5m } \\
\text { BC5m }\end{array}$ & $2,47 \pm 0,03 b$ & $2,64 \pm 0,03 a$ & $2,58 \pm 0,02 a$ & $2,57 \pm 0,03 a$ \\
\hline $\begin{array}{l}\mathrm{CC} 7 \mathrm{~m} \\
B C 7 \mathrm{~m}\end{array}$ & $2,57 \pm 0,03 a$ & $2,67 \pm 0,02 \mathrm{a}$ & $2,65 \pm 0,02 a$ & $2,60 \pm 0,03 a$ \\
\hline $\begin{array}{l}\mathrm{CC} 12 \mathrm{~m} \\
\mathrm{BC} 12 \mathrm{~m}\end{array}$ & $2,78 \pm 0,05 a$ & $2,79 \pm 0,04 a$ & $2,80 \pm 0,03 a$ & $2,74 \pm 0,05 a$ \\
\hline
\end{tabular}

leves ao nascer, seguidas das vacas velhas, sendo que as últimas produziram bezerros com peso ao nascer semelhante ao de vacas jovens e adultas.

Verifica-se, ainda, que os bezerros filhos de prímiparas continuaram mais leves até os 7 meses de idade, o que foi reflexo do menor GMD (Tabela 7) principalmente do nascimento até o desmame, e dos 84 dias aos 5 meses de idade, que foi mais longo, no qual o GMD foi numericamente inferior ao observado nos demais grupos de bezerros, o que está de acordo com o trabalho realizado por Nelson et al. (1982).

Na Tabela 9, encontram-se as médias para a CC dos bezerros aos 5, 7 e 12 meses de idade, de acordo com a classe de idade da vaca ao parto.

Aos 5 meses de idade, os bezerros filhos de prímiparas foram os que apresentaram menor CC, sendo que os demais grupos de bezerros não diferiram entre si. A pior CC dos bezerros filhos de prímiparas foi conseqüência da menor produção de leite dessas vacas, refletindo-se em menor GMD e peso dos bezerros nesta idade.

Aos 7 e 12 meses de idade, apesar de não ter sido observada diferença significativa para esta variável, ela ainda acompanhou o peso dos bezerros, sendo numericamente menor para os bezerros filhos de prímiparas em relação aos demais.

\section{Conclusões}

Bezerros desmamados aos 63 dias, cujas vacas foram suplementadas, foram mais pesados ao desmame e aos 84 dias.
Nos bezerros cujas vacas foram suplementadas, a redução na idade ao desmame de 63 para 42 dias resultou em menor peso aos 12 meses.

Bezerros mestiços foram mais pesados ao desmame que os puros, não diferindo dos Charolês aos 12 meses.

A idade da vaca influenciou o peso dos bezerros até os sete meses de idade.

\section{Literatura Citada}

ALBUQUerque, L.G.; ELER, J.P.; COSTA, M.J.R.P. et al. Produção de leite e desempenho do bezerro na fase de aleitamento em três raças bovinas de corte. Revista da Sociedade Brasileira de Zootecnia, v.22, n.5, p.745-754, 1993.

ALENCAR, M.M. Relação entre produção de leite da vaca e sesempenho do bezerro nas raças Canchim e Nelore. Revista da Sociedade Brasileira de Zootecnia, v.18, n.2, p.146-156, 1989.

CACHAPUZ, J.M.S. Desmame aos 90 dias: efeito da alimentação no desempenho de bezerros. Porto Alegre: EMATER, 1991. 32p.

CERDÓTES, L.; RESTLE, J.; ALVES FILHO, D.C. et al. Produção e composição do leite de vacas de quatro grupos genéticos submetidas a dois manejos alimentares no período de lactação. Revista Brasileira de Zootecnia, v.33, n.3. p.610-622, 2004.

HOUGHTON, P.L.; LEMENAGER, R.P.; HORSTMAN, L.A. Effects of body composition, pre and postpartum energy level and early weaning on reproductive performance o f beef cows and preweaning calf gain. Journal of Animal Science, v.68, p.1438-1446, 1990.

JENKINS, T.G.; CUNDIFF, L.V.; FERRELL, C.L. Diferences among breed crosses of cattle in the conversion of food energy to calf weight during the preweaning interval. Journal of Animal Science, v.69, p.2762-2769, 1991. 
LYFORD JR., S.J. Crecimiento y desarrollo del aparato digestivo de los rumiantes In: CHURCH, D.C. (Ed.) El rumiante fisiologia digestiva y nutrición. Zaragoza: Acribia, 1993. p.47-68.

MOOJEN, J.G.; RESTLE, J.; MOOJEN, E.L. et al. Efeito da época da desmama e da pastagem no desempenho de vacas e terneiros de corte. 2 - Desempenho de terneiros. Ciência Rural, v.24, n.2, p.399-403, 1994.

NELSON, L.A.; BEAVERS, G.D.; STEWART, T.S. Beef $x$ beef and dairy $x$ beef females mated to Angus and Charolais sires. II. Calf growth, weaning rate and cow productivity. Journal of Animal Science, v.54, n.6, p.1150-1159, 1982.

NEVILLE JR., W.E. Influence of dam's milk production and other factors on 120-day and 240-day weight of Hereford calves. Journal of Animal Science, v.21, p.315-320, 1962.

PASCOAL, L.L.; VAZ, F.N. Desmame precoce aos sessenta dias. In: RESTLE, J.; BRONDANI, I.L.; PASCOAL, L.L. et al. (Eds.) Técnicas avançadas na recria e engorda em bovinos de corte. Santa Maria: Universidade Federal de Santa Maria, 1997. p.36-50.

QUADROS, S.A.F.; LOBATO, J.F.P. Efeitos da lotação animal na produção de leite de vacas de corte primíparas e no desenvolvimento de seus bezerros. Revista Brasileira de Zootecnia, v.26, n.1, p.27-33, 1997.

RESTLE, J.; PACHECO, P.S.; PASCOAL, L.L. et al. Efeito da pastagem, da produção e composição do leite no desempenho de bezerros de diferentes grupos genéticos. Revista Brasileira de Zootecnia, v.33, n.3, p.691-703, 2004.

RESTLE, J.; POLLI, V.A.; ALVES FILHO, D.C. et al. Desenvolvimento de bovinos de corte de diferentes grupos genéticos desmamados aos 3 ou 7 meses de idade. Revista Brasileira de Zootecnia, v.28, n.5, p.1023-1030, 1999.

RESTLE, J.; VAZ, F.N. Desmame precoce de terneiros. In: SIMPÓSIO INTERNACIONAL SOBRE PRODUÇÃO INTENSIVA DE GADO DE CORTE, 2., 1998, São Paulo. Anais... São Paulo: 1998. p.3-9.
RIBEIRO, E.L.A.; RESTLE, J. Desempenho de terneiros Charolês e Aberdeen Angus puros e seus mestiços com Nelore. Pesquisa Agropecuária Brasileira, v.26, n.8, p.1145-1151, 1991.

ROBINSON, O.W.; YUSUFF, M.K.M.; DILLARD, E.U. Milk production in Hereford cows I. Means and correlations. Journal of Animal Science, v.47, n.1, p.131-136, 1978.

RUTLEDGE, J.J.; ROBISON, O.W.; AHLSCHWEDE, W.T. et al. Milk yield and its influence on 205-day weight of beef calves. Journal of Animal Science, v.33, n.3, p.563-567, 1971.

STATISTICAL ANALISYS SYSTEMS - SAS. User's guide: Version 6, Cary: 1993. 1042p.

SENNA, D.B. Desempenho reprodutivo e produção de leite de vacas de quatro grupos genéticos, desterneiradas precocemente, submetidas a diferentes períodos de pastagem cultivada. Santa Maria: Universidade Federal de Santa Maria, 1996, 85p. Dissertação (Mestrado em Zootecnia) - Universidade Federal de Santa Maria, 1996.

SIMEONE, A.; TRUJILLO, A.I.; CÓRDOBA, G. et al. Efecto del destete precoz y de dos sistemas de alimentacion postdestete sobre la ganancia de peso de terneros Hereford hasta los 15 meses de edad. Revista Argentina de Produção Animal, v.17, p.58-59, 1997.

STORY, C.E.; RASBY, R.J.; CLARK, R.T. et al. Age of calf at weaning of spring-calving beef cows and the effect on cow and calf performance and production economics. Journal of Animal Science, v.78, p.1403-1413, 2000. 